\title{
Taking Into Account Workers' Fatigue in Production Tasks: A Combined Simulation Framework
}

\author{
Aicha Ferjani $^{1} \quad$ Henri Pierreval $^{1} \quad$ Denis Gien $^{1} \quad$ Sabeur Elkosantini $^{2}$ \\ ${ }^{1}$ LIMOS, UMR 6158, SIGMA-Clermont, Aubière, France, \\ \{aicha.ferjani, henir.pierreval, denis.gien\} @sigma-clermont.fr \\ ${ }^{2}$ Industrial Engineering Department, College of Engineering Riyadh, Kingdom of Saudi Arabia, \\ Selkosantini@ksu.edu
}

\begin{abstract}
In manufacturing systems, workers are often subjected to arduous working conditions, such as heavy loads and discomfort postures, which induce fatigue. Because of the effect of fatigue on workers' well-being, as well as on their performances, managers would need to understand the evolution of operators' fatigue during their work, in order to make relevant decisions (e.g. work schedule, facility layout decisions, and rest periods). In this context, we present a simulation modeling framework to evaluate manufacturing systems, which takes the workers' fatigue into account. The suggested framework combines several worldviews: Discrete Event modeling, multi-agent and System Dynamics. Discrete Event concepts are used to describe the manufacturing system dynamic behavior and agents are used to model workers. One important characteristic of agents' behavior on which emphasis is put is fatigue, which is modeled using System Dynamics concepts. The proposed approach is implemented using the Anylogic simulation software.
\end{abstract}

Keywords: simulation discrete event, intelligent agents, system dynamics, manufacturing systems, fatigue

\section{Introduction}

Workers play a key role in modern manufacturing systems. They are often subjected to arduous working conditions, such as carrying heavy loads, noise and vibrations. This leads to fatigue in work, which causes a decline in workers' performance, errors and may lead to health troubles. As consequence, when they have to organize a manufacturing system, managers are also interested in management policies that can contribute to improve workers' performance, as well as their wellbeing at work. In this respect, managers need to understand the evolution of operators' fatigue during their work, depending on the organizational scenario considered for the manufacturing system. It has been widely demonstrated that fatigue in manufacturing depends on several factors, such as the penibility resulting from the production environment (e.g. noise), and the amount of work carried out by each operator. This amount of work depends on several operating conditions in the factory, such as the schedule of breaks and the penibility of the tasks that workers perform. The assignment of the workers to the different machines in the system has an effect on the work duration of each operator. The evolution of workers' fatigue depends on how they will be solicited, which can have different impacts, in terms of system performance (e.g. delay and quality). Therefore, it is important to determine the production scenarios that induce excessive fatigue, in order to improve workers' performances and well-being at work. As consequence, there is a need for dynamic models that allow the evolution of fatigue along time to be evaluated, as well as the manufacturing system performance under different production scenarios. For this purpose, simulation seems to be a relevant approach. However, how to build a suitable model of manufacturing system that takes fatigue into account turns out to be a difficult research question.

To address this question, we propose a simulation modeling framework to model manufacturing systems that can take workers' fatigue into account. The suggested framework combines the paradigms of Discrete Event Simulation (DES), System Dynamics (SD) and Intelligent Agents (IA) in the same simulation model. The DES concepts are used to describe the dynamic behavior of the manufacturing system. Workers are modeled as intelligent agents with a specific knowledge and behavior. Since fatigue represents an important characteristic of this behavior, we use SD concepts to describe the evolution of the operators' fatigue during the work. The suggested combined simulation framework is implemented using the Anylogic simulation software, which allows these different worldviews to be combined.

The remainder of this paper is organized as follows. Section 2 explains the novelties of this study with respect to the existing literature. Section 3 focuses on the proposed framework. In Section 4, a first implementation of the suggested approach using the Anylogic simulation software is presented. The final 
section draws the conclusions and some suggestions for future research.

\section{Related research}

In the last decades, researchers from different fields, such as the work psychology and ergonomics, have paid much attention to the fatigue phenomenon in manufacturing systems. In this context, few research works have used simulation to address such problems.

Digiesi et al. (2006 and 2009) have used simulation in order to investigate the impact of fatigue on the mean flowtime and the work in process in a manufacturing system. The authors have used DES to model a flow line system composed of one worker performing a repetitive task. However, fatigue is a continuous and a complex phenomenon that depends on several factors. For instance, Grandjean (1979) has highlighted that difficult tasks caused fatigue in manufacturing. Kahya (2007) has demonstrated that physical efforts and the penibility of the production environment contribute to increase the workers' fatigue.

Walters et al. (2000) have used DES to analyze the impact of fatigue and rotation schedules on workers performances. DES has also been used by Perez et al. (2014) to evaluate workers performances and cumulative spinal load in an assembly line. In these studies, the DES model of the manufacturing system is used to collect the data necessary (e.g. task durations, process time, and the availability of machines) for the work-rest models that they used to evaluate fatigue.

In the ergonomic literature, few approaches based on DES have been developed to help designers to understand the ergonomic impacts of a proposed alternative in system design. For example, Perez et al. (2014) have combined DES with a work-rest model to predict the effect of the mechanical exposure of workers and the accumulation of muscular fatigue. The authors have evaluated the accumulation of fatigue before and after performing a job such that, the time pattern of the cycle is given by the DES model of an assembly line. Thus, their model is not able to describe the evolution of fatigue during processing a task. Dode et al. (2015) have suggested an approach integrating fatigue-recovery pattern and learning into a DES model of an electronic assembly line to evaluate productivity and quality. The authors are interested in the muscular fatigue caused by the repetitive work. Therefore, they have evaluated fatigue through Muscular Endurance Time (MET) models, which are used when fatigue is assumed to be caused by the repetitive work.

According to Elkosantini and Gien (2009) workers are often assimilated to a simple resource with a failure rate, mean-time-between-failure and a repair time in most simulation models. However, workers, as human beings, exhibit a more complex and unpredictable behavior than the inanimate resources (Elkosantini and
Gien, 2007). Therefore, authors as Boudreau et al. (2003) have emphasized the importance of modeling the human behavior of workers.

In this respect, Elkosantini and Gien (2009) have suggested an Agent-based framework to model the human behavior including different behavioral aspects, such as stress and satisfaction, in order to analyze the behaviors of workers in manufacturing. The authors do not model the manufacturing system in their approach.

Fatigue represents an important characteristic of the workers' behavior. As mentioned above, it depends on several factors, which may be complex and interact with each other. Thus, it is difficult to describe how this phenomenon evolves along time. In order to deal with this problem, SD concepts seem to be relevant. The SD approach (Forrester, 1958), has been widely used to understand complex phenomenon in different fields but it seems not to be used for fatigue models.

In summarizing, DES has been used by few researchers to address very specific problems. Certain concepts, such as IA and SD can be used in order to better model workers in simulation so as to take their behavior and fatigue into account. Although different concepts can be combined in simulation (Elkosantini and Gien, 2009), to the best of our knowledge, no publication seems to combine the concept of DES, SD and IA together in the same simulation model so as to evaluate the impact of different production scenarios on both system performance and workers' fatigue.

\section{Related research Description of the proposed framework}

\subsection{Principle}

To evaluate the impacts of the production scenario on the system performance and the workers' fatigue, we need to a simulation model of the manufacturing system in which the workers are subjected to fatigue. To meet this objective, we propose to combine different worldviews in the same simulation model. The proposed simulation-modeling framework combines the following paradigms: IA, DES and SD. For further information about the combined simulation, we refer to the review of Dessouky and Roberts (1997).

The workers' fatigue depends on their activities. For instance, when the worker performs a tiring job, his/her fatigue increases. However, fatigue decreases when the worker is resting. This leads to a dynamic behavior of the worker. According to Elkosantini and Gien (2009), the workers exhibit a dynamic and more complex behavior than the inanimate resources. Therefore, modeling workers as inanimate resources, as they are often modeled in the existing simulation models, seems to be not relevant to take their behaviors into account.

As defined by Franklin and Graesser (1996), an agent "continuously performs three functions: perception of 
dynamic conditions in the environment; action to affect conditions in the environment; and reasoning to interpret perceptions, solve problems, draw inferences, and determine actions". According to Padgham and Lambrix (2000), the agent also shows a behavior. Based on the above definitions, we suggest using the concept of "Intelligent Agent" to model workers in our framework.

As mentioned in the related research, the behavior of workers in manufacturing is related to several aspects, such as the evolution of his/her fatigue or satisfaction and how to choose the tasks to be performed. These behavioral aspects are often complex. In order to describe the evolution of such behavioral aspects, we propose to use SD concepts, which are extensively used in the literature to describe the evolution of complex and continuous systems. Hence, a SD component describing how the worker behaves is integrated into the agent component of the model.

In order to describe how the worker behaves, we need to know several information about his/her work and the production environment. For instance, how many machines in the manufacturing and what is the physical effort required to work on each one. In addition, one needs to know the amount of work carried out by the agent to describe the impact of the accumulation of work on its fatigue. This requires information about the task completed, the work schedule and rest periods. This information is available thanks to the DES component of the manufacturing system model.

Figure 1 illustrates the global architecture of the suggested combined model. As it will be depicted below, the three components interact with each other.

\subsection{Discrete Event Simulation component}

The DES component represents the set of the elements of the manufacturing system (e.g. machines, products, transporters and stocks), which interact with the workers and, at the same time, have an impact on their fatigue. Thus, our DES component can describe the products, the manufacturing resources and several characteristics of the manufacturing system. The more classical performance criteria are computed in the DES component (e. g. mean flowtime of jobs (Neumann et al., 2006).

Typically, the machines in the system are modeled as resources, buffers are modeled as queues, etc. The penibility associated with the use of the machines has a great impact on the workers' fatigue. Thus, a coefficient Penibility of Machine is associated to each machine in the simulation model to describe the physical effort required by the worker to use that machine. We also need the position of each production facility since the distance walked by the operator also affects his/her fatigue. For that, each element in the DES component is characterized by its geographical coordinates.
The state variables describe the state of each element in the DES component. The dynamic behavior of the DES component has a significant impact on the amount of work that should be carried out by each worker. As consequence, the behavior of the system also affects the workers' fatigue. Therefore, we find that the state variables describing the state of each element in the DES component as well as, the events related to the start or the end of a production operation or transport activity, are used to evaluate the operators' fatigue.

As mentioned in the related research, the penibility of the production environment, such as the noise, temperature and vibrations, has a significant impact on the workers' fatigue. Thus, a subjective evaluation of the penibility of the production environment may be described, in our DES component, through the coefficient Penibility of Environment.

\subsection{Agent model}

As presented in Figure 1, we consider that the agent in our framework is characterized by:

- Goals: they can be classified in two types: the first type is common for all the agent, which is mainly related to the improvement of the performance of the manufacturing system, such as the minimization of the makespan or the balance of works between coworkers. The second type is specific to the agent such as the maximization of its satisfaction (regarding its preferences).

- Knowledge base: the agent has knowledge, which he/she uses in the manufacturing system. Among this knowledge, we can find the decision logic to select the set of machines on which the agent prefers to work. The agent has also knowledge about which assignment strategy should be selected so to achieve its goal.

- Capabilities: as mentioned in Franklin and Graesser (1996), the agent capabilities enable it to react rationally towards achieving a particular goal. Among these capabilities, we find the agent skills, which enable $\mathrm{him} / \mathrm{her}$, for example, to determine to which machine he/she can be assigned. The skill matrix can also contain the durations that may be needed by the agent on each machine. Based on whether the worker is novice or expert, these durations differ from one agent to another. In order to achieve its goals, the agent has physical capabilities such as the ability to recover after performing a job. For that, we consider the speed of recovery of the agent after performing a task, which varies according to the physical characteristics of the agent such as its age and gender.

- Communication: it represents his/her social network at work, (his/her acquaintance). The acquaintances can be the workers, which can collaborate with the agent. They can also include the supervisors. Thus, many types of communication may need to be established between agents. For instance, agents may 
negotiate to switch tasks if someone is exhausted. In order to establish communication, agents need a communication protocols such as the Contract Net Protocol (Sabar et al., 2009).

\subsection{System Dynamics component}

The SD component describes the evolution of the agent's fatigue according to his/her behavior during the

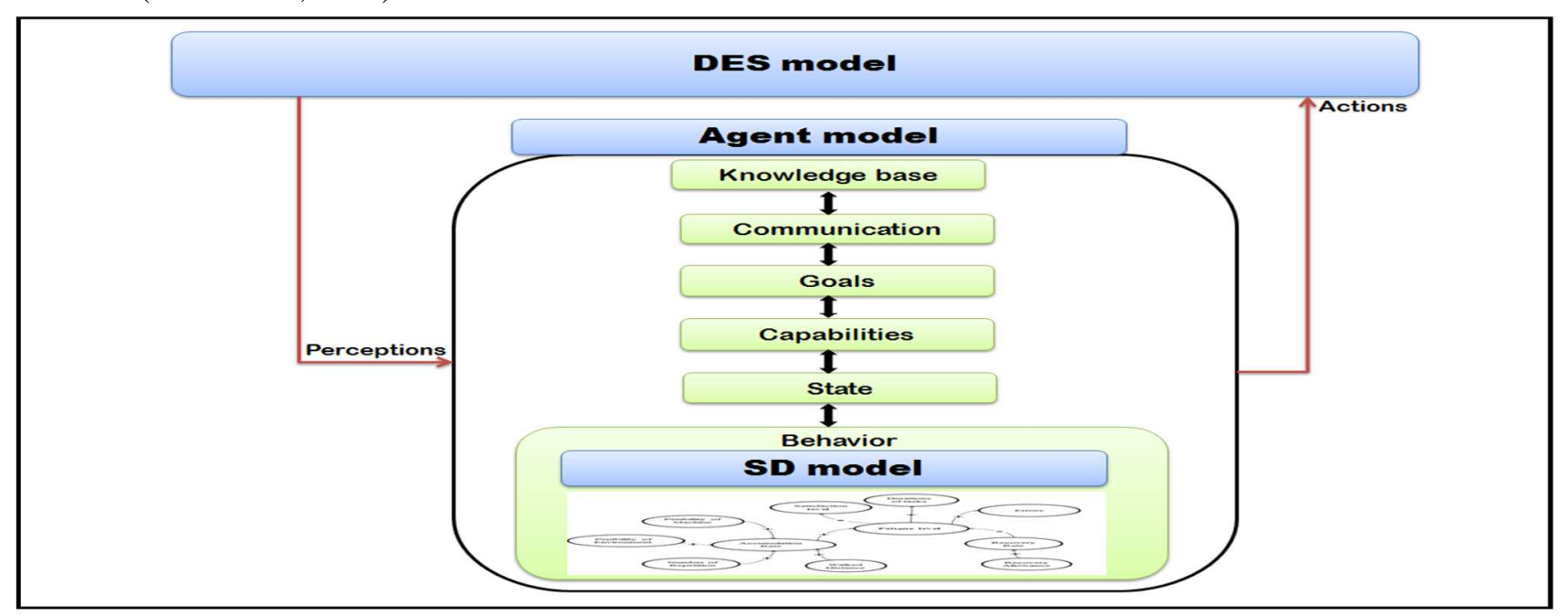

Figure 1. Overview of the proposed framework.

- State: in our model, the agent has different states: it can be idle, working, walking, resting and absent. State variables also describe the physical and psychological state of the agent such as Fatigue level and Satisfaction level, which indicate, respectively, the current level of fatigue and satisfaction of the worker.

- Perception: since we are in a simulation context, his/her perception of the environment (here the manufacturing systems and the other agents) is characterized: by the system state as it evolves in the DES component, and by what can be known about the other agents. For instance, the agent percepts the available machines in the DES model through the state variable Machine State, which is associated to each machine.

- Behavior: it describes the way how the state of an agent can change and how it can change the manufacturing system state. The behavior of the agent entails making some actions. For example, each agent can have assignment strategies to select to which machine he/she has to be assigned in order to satisfy his/her goal (e.g. to minimize the makespan). We can use several assignment rules, which are widely used in the literature, such as select the machine with Shortest Processing Time (SPT).

As mentioned in the related research, the behavior of workers in manufacturing is characterized by some behavioral aspects such as fatigue and satisfaction. This has an impact on how the behavioral aspects evolve along time. Let us take for example the fatigue of the agent: it increases during the period in which the agent is walking; however, not as much as when the agent is performing a tiring job. work. One of the objectives of the SD model is to describe the variation, over time, of the state variables associated with the behavioral aspects of the agent. In this study, we focus, in particular, on the evolution of the agent's fatigue.

Variations in the level of fatigue depend on several factors, such as the penibility associated with the use of machines. At the same time, these variations have a great impact on other state variables. For instance, the increase of Fatigue level leads to a decrease in Satisfaction level. As consequence, it is important to determine the causal relationships that may exist between the agent's fatigue, its factors and impacts. For that, we use a causal diagram. For illustration purposes, let us take the example of the causal diagram presented in Figure 2. This causal diagram is composed of a set of nodes, which represent the relationship between the relevant state variables used in the model.

The factors that induce fatigue are linked to the variable Fatigue Level by positive relations, since an increase of one factor causes an increase in the level of fatigue. For example, the worker's fatigue can increase with the distance he/she has walked until time t. For that, a positive relation connects the variable Walked Distance and Fatigue level. Repetitive activities also have an impact on fatigue. Therefore, there is a positive relationship connecting Fatigue level with the variable Number of Repetition, which indicates how many times the agent has repeated the job since the beginning of the simulation until time $t$.

The agent's fatigue has adverse effects on both the agent and DES model. These effects are represented by negative relations from the state variable Fatigue level. 
On the one hand, fatigue declines some agent capabilities. For instance, the increase of fatigue leads, according to Ferjani et al. (2015 and 2017), to an increase of the task durations. Based on the human behavioral model proposed by Elkosantini and Gien (2007), fatigue causes also a decrease in the worker satisfaction. Thus, an increase of the Fatigue level leads to a decrease in the Satisfaction level. On the other hand, fatigue causes errors so that deteriorates the quality of produced parts. This means that the number of defective parts in the DES model increases.

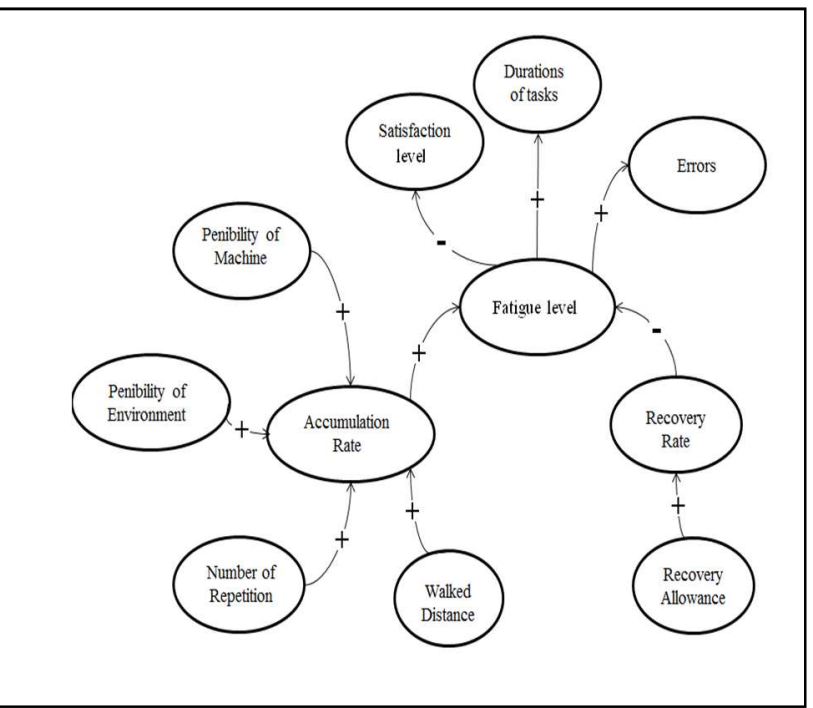

Figure 2. Causal diagram of the worker's fatigue.

As presented in Figure 2 and, according to Kahya (2007), the factors, which induce fatigue, control the speed of its accumulation. On the contrary, the recovery factors control the speed of the recovery. In our model, the parameter Recovery Allowance, which describes the physical capabilities of the agent, is used to control the speed of recovery. The variables Accumulation _Rate and Recovery_Rate control, respectively, the speed of accumulation and recovery of fatigue.

The variables in the nodes of the causal diagram (Figure 2) can be classified in two types: the first one is the discrete variables such as number of repetition of a task the worker has performed (Number of Repetitions). These variables change their value when events occur. The second type is the continuous variables such as Fatigue level and Satisfaction level. Their values change continuously, even when the agent is in the same state. Therefore, we use differential equations in order to describe the variations of the continuous variables over time. Since the way how fatigue evolves varies from one state to another, the differential equations, which are used to describe this evolution, vary also. Let us take, for example, the case where the agent is working. At this time, its fatigue grows in accordance with the penibility associated with the use of the machine (Penibility of Machine), the number of repetition of the task (Number of Repetition) and the penibility of the production environment (Penibility of Environment). In such case and based on the fatigue indicator proposed by Konz (1998), which is widely used in the ergonomic literature, the variation in the level of fatigue, when the agent is working, would be as follows:

$$
d F(t) / d t=A(e, p, r e p(t))(1-F(t))
$$

Where $F(t)$ is the current level of fatigue until time t. e and $\mathrm{p}$ represent, respectively, the coefficient Penibility of Environment and Penbility of Machine in the causal diagram of Figure 2. rep $(t)$ represents the number of repetition of the same task.

In the case where the agent is not working, its Fatigue level decreases. We assume that the recovery depends only on the parameter Recovery Allowance. Thus, the variation in the level of fatigue, during the rest period, can be as follows:

$$
d F(t) / d t=-R(r e c) F(t)
$$

Thus, the causal diagram, in Figure 2, can be translated into a flow-stock diagram. SD models are based mainly on stock (state) and flow (rate) variables. In our model, the stock variables represent the continuous state variables. For instance, the variable Fatigue level is considered as a stock variable since it describes the accumulation of fatigue during the work.

\section{Implementation with ANYLOGIC}

A first prototype simulation model with Anylogic has been developed to illustrate the proposed framework. The Anylogic simulation software, which is initially designed to support multiple modeling methods and their combinations (Borshchev, 2013), allows to use a combined simulation approach with several worldviews.

The different elements, which compose the DES component in our framework, can be represented using the Process Modeling Library of Anylogic, since this library supports the discrete-event modeling paradigms. The workers in the DES component are modeled as agents. For that, we represent them by a class Java since the Anylogic software is based on Java as a programming language.

Regarding the SD component, it is represented using the System Dynamics Palette of Anylogic. The state variable Fatigue level is represented by a stock, which is subjected to the variations in the flows (i.e. Accumulation Rate and Recovery Rate).

Our prototype is used to evaluate certain impacts of a given production scenarios on system performance for example, the flowtime of jobs in the system.

The evolution of the workers' fatigue along time can be described through curves, such as those presented in 
Figure 3. In Figure 3, we have four workers, so that each curve corresponds to the evolution of the fatigue of each one during $120 \mathrm{~min}$ of work. It can be noted that, during the period highlighted in Figure 3, the workers 1 and 2 are more tired than the workers 3 and 4 . It can also be seen that the worker 3 has been solicited more times than the worker 1 and 2. Even so, that worker is not tired as much as the workers 1 and 2 .

\section{Acknowledgements}

The authors are grateful to thank Dr. Vladimir Koltchanov, from Anylogic EUROPE, for his kind help. This project was partially supported by the CMCUUTIQUE program 14G 1412 and by SIGMA-Clermont. Their support is greatly appreciated.
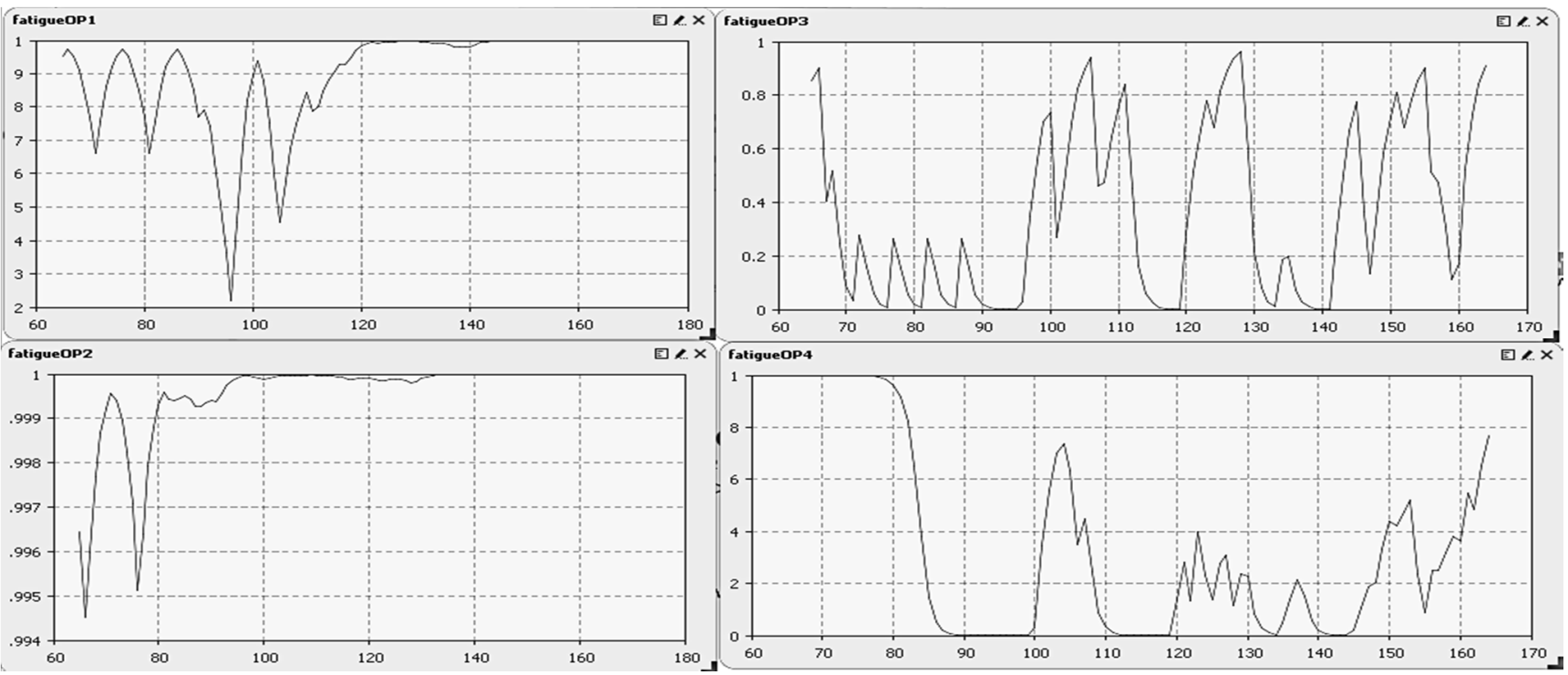

Figure 3. The evolution of workers' fatigue along time.

\section{Conclusions}

We have proposed a simulation modeling framework to evaluate manufacturing systems using conventional performance criteria, but also the workers' fatigue. The suggested framework combines three different paradigms (DES, SD and IA) in the same simulation model. DES is used to describe the different elements of the manufacturing system, the dynamic behavior of the system and to collect the resulting performance measures. The workers' behavior is taken into account through the use of IA concepts. The evolution of fatigue is described using SD concepts.

We have illustrated this combined approach using the ANYLOGIC simulation software. Simulation experiments show how the fatigue of each operator can evolve during his/her work depending on different organizational scenarios of the manufacturing systems. For instance, we can evaluate the impacts of different assignment strategies on workers' fatigue or of different layout of the facilities.

In order to determine the differential equations, which describe the evolution of fatigue in each state, we are based here on an indicator of fatigue (Konz, 1998), which is widely used in the literature. However, the proposed equations can be adapted with other indicators of fatigue without changing the key principles of our framework.

\section{References}

A. Borshchev. The big book of simulation modeling: multimethod modeling with AnyLogic 6, AnyLogic North America, 2013.

A. Ferjani, A. Ammar, H. Pierreval, and A. Trabelsi. A Heuristic approach taking operators' fatigue into account for the dynamic assignment of workforce to reduce the mean flowtime, In International Conference on Computers and Industrial Engineering, CIE45, 2015.

A. Ferjani, A. Ammar, H. Pierreval, and S. Elkosantini. A simulation-optimization based heuristic for the online assignment of multi-skilled workers subjected to fatigue in manufacturing systems, Comput. Ind. Eng, 112: 663674, 2017.

B. Walters, J. French, and J. Barnes. Michael. Modeling the effects of crew size and crew fatigue on the control of tactical unmanned aerial vehicles (TUAVs), In Simulation Conference, Proceedings, 2000.

D. Dessouky and C. A. Roberts. A Review and Classification of Combined Simulation, Comput. Ind. Eng., 32 (2): 251-264, 1997.

E. Kahya. The effects of job characteristics and working conditions on job performance, Int. J. Ind. Ergon., 37 (6): 515-523, 2007.

G. Grandjean. Fatigue in industry, Br. J. Ind. Med., 36 (3): 175-186, 1979.

J. Boudreau, W. Hopp, J. O. McClain, and L. Joseph Thomas. On the interface between operations and human resources 
management, Manuf. Serv. Oper. Manag., 5 (3), 179-202, 2003.

J. Perez, M. P. De Looze, T. Bosch, and W. P. Neumann. Discrete event simulation as an ergonomic tool to predict workload exposures during systems design, Int. J. Ind. Ergon., 44 (2): 298-306, 2014.

J. W. Forrester. Industrial dynamics: a major breakthrough for decision makers, Harv. Bus. Rev., 36 (4): 37-66, 1958.

L. Padgham and P. Lambrix. Agent Capabilities: Extending BDI Theory, In Proc. Seventeenth Natl. Conf. Artif. Intell. AAAI, 2000.

M. Sabar, B. Montreuil, and J.-M. Frayret. A multiagentbased approach for personnel scheduling in assembly centers, Eng. Appl. Artif. Intell, 22 (7): 1080-1088, 2009.

P. Dode, M. Greig, S. Zolfaghari, and W. P. Neumann. Integrating Human Factors into Discrete Event Simulation: A proactive approach to simultaneously design for system performance and employees' well being, Int. J. Prod. Res., 54 (10): 3105-3117, 2015.

S. Digiesi, G. Mossa, G. Mummolo, and P. Bari. Performance measurement and 'Personnel-Oriented' simulation of an assembly line, In Proceedings of AMS, 2006.

S. Digiesi, A. a. a. Kock, G. Mummolo, and J. E. Rooda. The effect of dynamic worker behavior on flow line performance, Int. J. Prod. Econ., 120 (2): 368-377, 2009.

S. Elkosantini and D. Gien. Human Behavior and Social Network Simulation : Fuzzy Sets / Logic And AgentsBased Approach, Soc. Networks, 1: 102-109, 2007.

S. Elkosantini and D. Gien. Integration of human behavioural aspects in a dynamic model for a manufacturing system, Int. J. Prod. Res., 47 (10): 2601-2623, 2009.

S. Franklin and A. Graesser. Is it an Agent, or just a program? : a taxonomy for autonomous agents, In Intelligent agents III agent theories, architectures, and languages, Springer, 1996.

S. Konz. Work/rest: Part II - The scientific basis (knowledge base) for the guide, Int. J. Ind. Ergon., 22 (1-2): 73-99, 1998.

T. Parsons. Politics and Social Structures, Free Press. New York, 1979.

W. P. Neumann, W. Jörgen, R. M. L. Medbo, and S. E. Mathiassen. Production system design elements influencing productivity and ergonomics: A case study of parallel and serial flow strategies, Int. J. Oper. Prod. Manag, 26 (8): 904-923, 2006. 